PROCEEDINGS OF THE

AMERICAN MATHEMATICAL SOCIETY

Volume 132, Number 4, Pages 981-986

S 0002-9939(03)07177-6

Article electronically published on November 4, 2003

\title{
SOME NUMERICAL INVARIANTS OF LOCAL RINGS
}

\author{
JOSEP ÀLVAREZ MONTANER
}

(Communicated by Bernd Ulrich)

\begin{abstract}
Let $R$ be a formal power series ring over a field of characteristic zero and $I \subseteq R$ any ideal. The aim of this work is to introduce some numerical invariants of the local rings $R / I$ by using the theory of algebraic $\mathcal{D}$-modules. More precisely, we will prove that the multiplicities of the characteristic cycle of the local cohomology modules $H_{I}^{n-i}(R)$ and $H_{\mathfrak{p}}^{p}\left(H_{I}^{n-i}(R)\right)$, where $\mathfrak{p} \subseteq R$ is any prime ideal that contains $I$, are invariants of $R / I$.
\end{abstract}

\section{INTRODUCTION}

Let $(R, \mathfrak{m}, k)$ be a regular local ring of dimension $n$ containing the field $k$, and let $A$ be a local ring that admits a surjective ring homomorphism $\pi: R \longrightarrow A$. Set $I=\operatorname{Ker} \pi$. G. Lyubeznik [9] defines a new set of numerical invariants of $A$ by means of the Bass numbers $\lambda_{p, i}(A):=\mu_{p}\left(\mathfrak{m}, H_{I}^{n-i}(R)\right):=\operatorname{dim}_{k} \operatorname{Ext}_{R}^{p}\left(k, H_{I}^{n-i}(R)\right)$. This invariant depends only on $A, i$ and $p$, but neither on $R$ nor on $\pi$. Completion does not change $\lambda_{p, i}(A)$; so one can assume $R=k\left[\left[x_{1}, \ldots, x_{n}\right]\right]$, with $x_{1}, \ldots, x_{n}$ independent variables.

Lyubeznik numbers can be described as the multiplicities of the characteristic cycle of the local cohomology modules $H_{\mathfrak{m}}^{p}\left(H_{I}^{n-i}(R)\right)$. The aim of this work is to prove that the multiplicities of the characteristic cycle of the local cohomology modules $H_{I}^{n-i}(R)$ and $H_{\mathfrak{p}}^{p}\left(H_{I}^{n-i}(R)\right)$, where $\mathfrak{p} \subseteq R$ is any prime ideal that contains $I$, are also invariants of $R / I$. Among these invariants we may find the Bass numbers $\mu_{p}\left(\mathfrak{p}, H_{I}^{n-i}(R)\right):=\operatorname{dim}_{k(\mathfrak{p})} \operatorname{Ext}_{R_{\mathfrak{p}}}^{p}\left(k(\mathfrak{p}), H_{I R_{\mathfrak{p}}}^{n-i}\left(R_{\mathfrak{p}}\right)\right)$

\section{The CHARACTERISTIC CYCLE}

In the sequel, $\mathcal{D}$ will denote the ring of differential operators corresponding to the formal power series ring $R=k\left[\left[x_{1}, \ldots, x_{n}\right]\right]$, where $k$ is a field of characteristic zero and $x_{1}, \ldots, x_{n}$ are independent variables. For details we refer to [4] and [5]. The ring $\mathcal{D}$ has a natural increasing filtration given by the order such that the corresponding associated graded ring $\operatorname{gr}(\mathcal{D})$ is isomorphic to the polynomial ring $R\left[\xi_{1}, \ldots, \xi_{n}\right]$.

Let $M$ be a finitely generated $\mathcal{D}$-module equipped with a good filtration, i.e., an increasing sequence of finitely generated $R$-submodules such that the associated graded module $\operatorname{gr}(M)$ is a finitely generated $\operatorname{gr}(\mathcal{D})$-module. The characteristic ideal of $M$ is the ideal in $\operatorname{gr}(\mathcal{D})=R\left[\xi_{1}, \ldots, \xi_{n}\right]$ given by $J(M):=$

Received by the editors September 24, 2002 and, in revised form, December 2, 2002.

2000 Mathematics Subject Classification. Primary 13D45, 13N10.

Key words and phrases. Local cohomology, $\mathcal{D}$-modules. 
$\operatorname{rad}\left(\operatorname{Ann}_{\operatorname{gr}(\mathcal{D})}(\operatorname{gr}(M))\right)$. One may prove that $J(M)$ is independent of the good filtration on $M$. The characteristic variety of $M$ is the closed algebraic set given by

$$
C(M):=V(J(M)) \subseteq \operatorname{Spec}(\operatorname{gr}(\mathcal{D}))=\operatorname{Spec}\left(R\left[\xi_{1}, \ldots, \xi_{n}\right]\right) .
$$

The characteristic variety allows us to describe the support of a finitely generated $\mathcal{D}$-module as an $R$-module. Let $\pi: \operatorname{Spec}\left(R\left[\xi_{1}, \ldots, \xi_{n}\right]\right) \longrightarrow \operatorname{Spec}(R)$ be the map defined by $\pi(x, \xi)=x$. Then $\operatorname{Supp}_{R}(M)=\pi(C(M))$.

The characteristic cycle of $M$ is defined as

$$
C C(M)=\sum m_{i} V_{i}
$$

where the sum is taken over all the irreducible components $V_{i}=V\left(\mathfrak{q}_{i}\right)$ of the characteristic variety $C(M)$, where $\mathfrak{q}_{i} \in \operatorname{Spec}(\operatorname{gr}(\mathcal{D}))$ and $m_{i}$ is the multiplicity of the module $\operatorname{gr}(M)_{\mathfrak{q}_{i}}$. Notice that the contraction of $\mathfrak{q}_{i}$ to $R$ is a prime ideal; so the variety $\pi\left(V_{i}\right)$ is irreducible.

2.1. Bass numbers and characteristic cycle. Let $\mathfrak{p} \in \operatorname{Spec}(R)$ be a prime ideal. The Bass numbers $\mu_{p}\left(\mathfrak{p}, H_{I}^{n-i}(R)\right)$ of the local cohomology modules $H_{I}^{n-i}(R)$, where $I \subseteq R$ is any ideal, can be described as the multiplicities of the characteristic cycle of $H_{\mathfrak{p}}^{p}\left(H_{I}^{n-i}(R)\right)$. Namely, we have:

Proposition 2.1. Let $I \subseteq R$ be an ideal, $\mathfrak{p} \subseteq R$ a prime ideal, and let

$$
C C\left(H_{\mathfrak{p}}^{p}\left(H_{I}^{n-i}(R)\right)\right)=\sum \lambda_{\mathfrak{p}, p, i, \alpha} V_{\alpha}
$$

be the characteristic cycle of the local cohomology module $H_{\mathfrak{p}}^{p}\left(H_{I}^{n-i}(R)\right)$. Then, the Bass numbers with respect to $\mathfrak{p}$ of $H_{I}^{n-i}(R)$ are

$$
\mu_{p}\left(\mathfrak{p}, H_{I}^{n-i}(R)\right)=\lambda_{\mathfrak{p}, p, i, \alpha_{\mathfrak{p}}}
$$

where $\pi\left(V_{\alpha_{\mathfrak{p}}}\right)$ is the subvariety of $X=\operatorname{Spec}(R)$ defined by $\mathfrak{p}$.

Proof. Let $\widehat{R_{\mathfrak{p}}}$ be the completion with respect to the maximal ideal $\mathfrak{p} R_{\mathfrak{p}}$ of the localization $R_{\mathfrak{p}}$. Notice that $\widehat{R_{\mathfrak{p}}}$ is a formal power series ring of dimension ht $\mathfrak{p}$. Since Bass numbers are invariant by completion we have

$$
\mu_{p}\left(\mathfrak{p}, H_{I}^{n-i}(R)\right)=\mu_{p}\left(\mathfrak{p} \widehat{R_{\mathfrak{p}}}, H_{I \widehat{R_{\mathfrak{p}}}}^{n-i}\left(\widehat{R_{\mathfrak{p}}}\right)\right)=\mu_{0}\left(\mathfrak{p} \widehat{R_{\mathfrak{p}}}, H_{\mathfrak{p} \widehat{R_{\mathfrak{p}}}}^{p}\left(H_{I \widehat{R_{\mathfrak{p}}}}^{n-i}\left(\widehat{R_{\mathfrak{p}}}\right)\right)\right)
$$

where the last assertion follows from [9, Lemma 1.4]. By using [9, Theorem 3.4], we have

$$
H_{\mathfrak{p} \widehat{R_{\mathfrak{p}}}}^{p}\left(H_{I \widehat{R_{\mathfrak{p}}}}^{n-i}\left(\widehat{R_{\mathfrak{p}}}\right)\right)=E_{\widehat{R_{\mathfrak{p}}}}\left(\widehat{R_{\mathfrak{p}}} / \mathfrak{p} \widehat{R_{\mathfrak{p}}}\right)^{\mu_{0}\left(\mathfrak{p} \widehat{R_{\mathfrak{p}}}, H_{\mathfrak{p} \widehat{R_{\mathfrak{p}}}}^{p}\left(H_{I \widehat{R_{\mathfrak{p}}}}^{n-i}\left(\widehat{R_{\mathfrak{p}}}\right)\right)\right)}
$$

So, its characteristic cycle is

$$
C C\left(H_{\mathfrak{p} \widehat{R_{\mathfrak{p}}}}^{p}\left(H_{I \widehat{R_{\mathfrak{p}}}}^{n-i}\left(\widehat{R_{\mathfrak{p}}}\right)\right)\right)=\mu_{p}\left(\mathfrak{p}, H_{I}^{n-i}(R)\right) V_{\alpha_{\mathfrak{p}}}^{\prime},
$$

where $\pi\left(V_{\alpha_{\mathfrak{p}}}^{\prime}\right)$ is the subvariety of $X^{\prime}=\operatorname{Spec} \widehat{R_{\mathfrak{p}}}$ defined by the ideal $\mathfrak{p} \widehat{R_{\mathfrak{p}}}$. Notice that we have used the following fact (see [9] and [1] for details):

$$
C C\left(H_{\mathfrak{p} \widehat{R_{\mathfrak{p}}}}^{\mathrm{ht} \mathfrak{p}}\left(\widehat{R_{\mathfrak{p}}}\right)\right)=C C\left(E_{\widehat{R_{\mathfrak{p}}}}\left(\widehat{R_{\mathfrak{p}}} / \mathfrak{p} \widehat{R_{\mathfrak{p}}}\right)\right)=V_{\alpha_{\mathfrak{p}}}^{\prime}
$$

Finally, by using the flatness of the morphism $R \longrightarrow \widehat{R_{\mathfrak{p}}}$, this characteristic cycle can be obtained from the characteristic cycle of $H_{\mathfrak{p}}^{p}\left(H_{I}^{n-i}(R)\right)$. Namely, if

$$
C C\left(H_{\mathfrak{p}}^{p}\left(H_{I}^{n-i}(R)\right)\right)=\sum \lambda_{\mathfrak{p}, p, i, \alpha} V_{\alpha}
$$


is the characteristic cycle of the module $H_{\mathfrak{p}}^{p}\left(H_{I}^{n-i}(R)\right)$, then we have

$$
C C\left(H_{\mathfrak{p} \widehat{R_{\mathfrak{p}}}}^{p}\left(H_{I \widehat{R_{\mathfrak{p}}}}^{n-i}\left(\widehat{R_{\mathfrak{p}}}\right)\right)\right)=\lambda_{\mathfrak{p}, p, i, \alpha_{\mathfrak{p}}} V_{\alpha_{\mathfrak{p}}}^{\prime} .
$$

2.2. Direct image. Some geometrical operations as the direct image have a key role in the theory of $\mathcal{D}$-modules. Our aim in this section is to give a brief survey of this operation in the particular case of the injection of $\mathbb{A}_{k}^{n}$ in $\mathbb{A}_{k}^{n+1}$. The main references we will use in this section are [5] and [10].

Let $\mathcal{D}_{n+1}$ and $\mathcal{D}_{n}$ be the rings of differential operators corresponding to $R^{\prime}=$ $k\left[\left[x_{1}, \ldots, x_{n}, t\right]\right]$ and $R=k\left[\left[x_{1}, \ldots, x_{n}\right]\right]$, respectively. Let $M$ be a $\mathcal{D}_{n}$-module. The direct image corresponding to the injection is the $\mathcal{D}_{n+1}$-module $i_{+}(M)$ defined as

$$
i_{+}(M)=k\left[\partial_{t}\right] \widehat{\otimes}_{k} M=M\left[\partial_{t}\right] .
$$

The characteristic variety of $i_{+}(M)$ can be computed from the characteristic variety of $M$. Namely, we have

$$
C\left(i_{+}(M)\right)=\{(\mathbf{x}, 0, \xi, \tau) \mid(\mathbf{x}, \xi) \in C(M)\} \subseteq \operatorname{Spec}\left(R^{\prime}\left[\xi_{1}, \ldots, \xi_{n}, \tau\right]\right),
$$

where we have considered $C(M) \subseteq \operatorname{Spec}\left(R\left[\xi_{1}, \ldots, \xi_{n}\right]\right)$.

The direct image of local cohomology modules can be easily described. The following result is stated as is used in our work.

Lemma 2.2. Let $\mathfrak{p} \subseteq R$ be a prime ideal that contains an ideal $I \subseteq R$. The direct image of the local cohomology module $H_{\mathfrak{p}}^{p}\left(H_{I}^{n-i}(R)\right)$ is

$$
i_{+}\left(H_{\mathfrak{p}}^{p}\left(H_{I}^{n-i}(R)\right)\right)=H_{(t)}^{1}\left(H_{\mathfrak{p} R^{\prime}}^{p}\left(H_{I R^{\prime}}^{n-i}\left(R^{\prime}\right)\right)\right) .
$$

Proof. Let $\mathcal{D}_{t}$ be the ring of differential operators corresponding to the formal power series ring $k[[t]]$. For simplicity we will denote the local cohomology modules $H_{\mathfrak{p}}^{p}\left(H_{I}^{n-i}(R)\right)$ and $H_{\mathfrak{p} R^{\prime}}^{p}\left(H_{I R^{\prime}}^{n-i}\left(R^{\prime}\right)\right)$ by $N$ and $N^{\prime}$, respectively. Then we have

$$
H_{(t)}^{1}\left(N^{\prime}\right)=H_{(t)}^{1}\left(N \hat{\otimes}_{k} k[[t]]\right)=H_{(t)}^{1}(k[[t]]) \hat{\otimes}_{k} N=\left(\mathcal{D}_{t} / \mathcal{D}_{t} \cdot(t)\right) \hat{\otimes}_{k} N=i_{+}(N) .
$$

Remark 2.3. In general, let $I_{1}, \ldots, I_{s}$ be a set of ideals of $R$. Then, the direct image of the local cohomology module $H_{I_{1}}^{i_{1}}\left(\cdots\left(H_{I_{s}}^{i_{s}}(R)\right) \cdots\right)$ is

$$
i_{+}\left(H_{I_{1}}^{i_{1}}\left(\cdots\left(H_{I_{s}}^{i_{s}}(R)\right) \cdots\right)\right)=H_{(t)}^{1}\left(H_{I_{1} R^{\prime}}^{i_{1}}\left(\cdots\left(H_{I_{s} R^{\prime}}^{i_{s}}\left(R^{\prime}\right)\right) \cdots\right)\right) .
$$

\section{Multiplicities of the Characteristic CyCle}

Let $A$ be a ring that admits a presentation $A \cong R / I$ for a given ideal $I \subseteq$ $R=k\left[\left[x_{1}, \ldots, x_{n}\right]\right]$. Recall that we have $\operatorname{Spec}(A)=\{\mathfrak{p} \in \operatorname{Spec}(R) \mid I \subseteq \mathfrak{p}\}$. Throughout this section, a prime ideal of $A$ will also mean the corresponding prime ideal of $R$ that contains $I$.

Let $R / I$ and $R^{\prime} / I^{\prime}$ be two different presentations of the local ring $A$. Then, for any prime ideal of $A$, we will denote $\mathfrak{p}^{\prime} \in \operatorname{Spec}\left(R^{\prime}\right)$ to be the prime ideal that corresponds to $\mathfrak{p} \in \operatorname{Spec}(R)$ by the isomorphism $\operatorname{Spec}(R / I) \cong \operatorname{Spec}\left(R^{\prime} / I^{\prime}\right)$.

Theorem 3.1. Let $A$ be a local ring that admits a surjective ring homomorphism $\pi: R \longrightarrow A$, where $R=k\left[\left[x_{1}, \ldots, x_{n}\right]\right]$ is the formal power series ring. Set $I=$ $\operatorname{ker} \pi$, let $\mathfrak{p} \subseteq A$ be a prime ideal and let

$$
C C\left(H_{\mathfrak{p}}^{p}\left(H_{I}^{n-i}(R)\right)\right)=\sum \lambda_{\mathfrak{p}, p, i, \alpha} V_{\alpha}
$$


be the characteristic cycle of the local cohomology modules $H_{\mathfrak{p}}^{p}\left(H_{I}^{n-i}(R)\right)$. Then the multiplicities $\lambda_{\mathfrak{p}, p, i, \alpha}$ depend only on $A, \mathfrak{p}, p, i$ and $\alpha$ but not on $R$ or $\pi$.

The proof of the theorem is inspired in the proof of [9] Theorem 4.1], but here we must be careful with the behavior of the characteristic cycle. So instead of [9] Lemma 4.3] we will use the following:

Lemma 3.2. Let $g: R^{\prime} \longrightarrow R$ be a surjective ring homomorphism, where $R^{\prime}$ is a formal power series ring of dimension $n^{\prime}$. Set $I^{\prime}=\operatorname{ker} \pi g$ and let

$$
C C\left(H_{\mathfrak{p}}^{p}\left(H_{I}^{n-i}(R)\right)\right)=\sum \lambda_{\mathfrak{p}, p, i, \alpha} V_{\alpha}
$$

be the characteristic cycle of the local cohomology modules $H_{\mathfrak{p}}^{p}\left(H_{I}^{n-i}(R)\right)$. Then, the characteristic cycle of $H_{\mathfrak{p}^{\prime}}^{p}\left(H_{I^{\prime}}^{n^{\prime}-i}\left(R^{\prime}\right)\right)$ is

$$
C C\left(H_{\mathfrak{p}^{\prime}}^{p}\left(H_{I^{\prime}}^{n^{\prime}-i}\left(R^{\prime}\right)\right)\right)=\sum \lambda_{\mathfrak{p}, p, i, \alpha} V_{\alpha}^{\prime},
$$

where $\pi\left(V_{\alpha}^{\prime}\right)$ is the subvariety of $X^{\prime}=\operatorname{Spec} R^{\prime}$ defined by the defining ideal of $\pi\left(V_{\alpha}\right)$ contracted to $R^{\prime}$.

Proof. $R$ is regular, and so Ker $g$ is generated by $n^{\prime}-n$ elements that form part of a minimal system of generators of the maximal ideal $\mathfrak{m}^{\prime} \subseteq R^{\prime}$. By induction on $n^{\prime}-n$ we are reduced to the case $n^{\prime}-n=1$. So Ker $g$ is generated by one element $f \in \mathfrak{m}^{\prime} \backslash \mathfrak{m}^{\prime 2}$. By Cohen's structure theorem, $R^{\prime}=k\left[\left[x_{1}, \ldots, x_{n}, t\right]\right]$ where we assume $f=t$ by a change of variables. We identify $R$ with the subring $k\left[\left[x_{1}, \ldots, x_{n}\right]\right]$ of $R^{\prime}$. In particular, we have to consider $I^{\prime}=I R^{\prime}+(t)$ and $\mathfrak{p}^{\prime}=\mathfrak{p} R^{\prime}+(t)$.

By using Lemma 2.2 and the degeneration of Grothendieck's spectral sequence $E_{2}^{p, q}=H_{(t)}^{p}\left(H_{J}^{q}(M)\right) \Longrightarrow H_{J+(t)}^{p+q}(M)$ we have

$$
\begin{aligned}
i_{+}\left(H_{\mathfrak{p}}^{p}\left(H_{I}^{n-i}(R)\right)\right) & =H_{(t)}^{1}\left(H_{\mathfrak{p} R^{\prime}}^{p}\left(H_{I R^{\prime}}^{n-i}\left(R^{\prime}\right)\right)\right)=H_{\mathfrak{p} R^{\prime}}^{p}\left(H_{(t)}^{1}\left(H_{I R^{\prime}}^{n-i}\left(R^{\prime}\right)\right)\right) \\
& =H_{\mathfrak{p} R^{\prime}}^{p}\left(H_{I R^{\prime}+(t)}^{n+1-i}\left(R^{\prime}\right)\right)=H_{\mathfrak{p} R^{\prime}}^{p}\left(H_{I^{\prime}}^{n^{\prime}-i}\left(R^{\prime}\right)\right) \\
& =H_{\mathfrak{p} R^{\prime}+(t)}^{p}\left(H_{I^{\prime}}^{n^{\prime}-i}\left(R^{\prime}\right)\right)=H_{\mathfrak{p}^{\prime}}^{p}\left(H_{I^{\prime}}^{n^{\prime}-i}\left(R^{\prime}\right)\right),
\end{aligned}
$$

where the second to last assertion comes from the fact that $H_{I^{\prime}}^{n^{\prime}-i}\left(R^{\prime}\right)$ is a $(t)$-torsion module. Then we are done by the results in Section 2.2.

Now we continue the proof of Theorem 3.1 .

Proof. Let $\pi^{\prime}: R^{\prime} \longrightarrow A$ and $\pi^{\prime \prime}: R^{\prime \prime} \longrightarrow A$ be surjections with $R^{\prime}=k\left[\left[y_{1}, \ldots, y_{n^{\prime}}\right]\right]$ and $R^{\prime \prime}=k\left[\left[z_{1}, \ldots, z_{n^{\prime \prime}}\right]\right]$. Let $I^{\prime}=\operatorname{ker} \pi^{\prime}$ and let $I^{\prime \prime}=\operatorname{ker} \pi^{\prime \prime}$. Let $R^{\prime \prime \prime}=R^{\prime} \widehat{\otimes}_{k} R^{\prime \prime}$ be the external tensor product, $\pi^{\prime \prime \prime}=\pi^{\prime} \widehat{\otimes}_{k} \pi^{\prime \prime}: R^{\prime} \widehat{\otimes}_{k} R^{\prime \prime} \longrightarrow A$ and $I^{\prime \prime \prime}=\operatorname{ker} \pi^{\prime \prime \prime}$.

By Lemma 3.2. if the characteristic cycle of $H_{\mathfrak{p}^{\prime}}^{p}\left(H_{I^{\prime}}^{n^{\prime}-i}\left(R^{\prime}\right)\right)$ is

$$
C C\left(H_{\mathfrak{p}^{\prime}}^{p}\left(H_{I^{\prime}}^{n^{\prime}-i}\left(R^{\prime}\right)\right)\right)=\sum \lambda_{\mathfrak{p}, p, i, \alpha}^{\prime} V_{\alpha}^{\prime}
$$

then the characteristic cycle of $H_{\mathfrak{p}^{\prime \prime \prime}}^{p}\left(H_{I^{\prime \prime \prime}}^{n^{\prime}+n^{\prime \prime}-i}\left(R^{\prime \prime \prime}\right)\right)$ is

$$
C C\left(H_{\mathfrak{p}^{\prime \prime \prime}}^{p}\left(H_{I^{\prime \prime \prime}}^{n^{\prime}+n^{\prime \prime}-i}\left(R^{\prime}\right)\right)\right)=\sum \lambda_{\mathfrak{p}, p, i, \alpha}^{\prime} V_{\alpha}^{\prime \prime \prime},
$$

where $\pi\left(V_{\alpha}^{\prime \prime \prime}\right)$ is the subvariety of $X^{\prime \prime \prime}=\operatorname{Spec} R^{\prime \prime \prime}$ defined by the defining ideal of $\pi\left(V_{\alpha}^{\prime}\right)$ contracted to $R^{\prime \prime \prime}$.

By Lemma 3.2. if the characteristic cycle of $H_{\mathfrak{p}^{\prime \prime}}^{p}\left(H_{I^{\prime \prime}}^{n^{\prime \prime}-i}\left(R^{\prime \prime}\right)\right)$ is

$$
C C\left(H_{\mathfrak{p}^{\prime \prime}}^{p}\left(H_{I^{\prime \prime}}^{n^{\prime \prime}-i}\left(R^{\prime \prime}\right)\right)\right)=\sum \lambda_{\mathfrak{p}, p, i, \alpha}^{\prime \prime} V_{\alpha}^{\prime \prime}
$$


then the characteristic cycle of $H_{\mathfrak{p}^{\prime \prime \prime}}^{p}\left(H_{I^{\prime \prime \prime}}^{n^{\prime}+n^{\prime \prime}-i}\left(R^{\prime \prime \prime}\right)\right)$ is

$$
C C\left(H_{\mathfrak{p}^{\prime \prime \prime}}^{p}\left(H_{I^{\prime \prime \prime}}^{n^{\prime}+n^{\prime \prime}-i}\left(R^{\prime}\right)\right)\right)=\sum \lambda_{\mathfrak{p}, p, i, \alpha}^{\prime \prime} V_{\alpha}^{\prime \prime \prime}
$$

where $\pi\left(V_{\alpha}^{\prime \prime \prime}\right)$ is the subvariety of $X^{\prime \prime \prime}=\operatorname{Spec} R^{\prime \prime \prime}$ defined by the defining ideal of $\pi\left(V_{\alpha}^{\prime \prime}\right)$ contracted to $R^{\prime \prime \prime}$.

In particular, we have $\lambda_{\mathfrak{p}, p, i, \alpha}^{\prime}=\lambda_{\mathfrak{p}, p, i, \alpha}^{\prime \prime}$ for all $\mathfrak{p}, p, i$ and $\alpha$.

Remark 3.3. By the same arguments one may prove that the multiplicities of the characteristic cycle of the local cohomology modules $H_{I_{1}}^{i_{1}}\left(\cdots\left(H_{I_{s}}^{i_{s}}(R)\right) \cdots\right)$, where $I_{1}, \ldots, I_{s}$ is a set of ideals of $R$ containing the ideal $I=I_{s}$, are also invariants of $R / I$.

Since Bass numbers $\mu_{p}\left(\mathfrak{p}, H_{I}^{n-i}(R)\right)$ are multiplicities of the characteristic cycle of $H_{\mathfrak{p}}^{p}\left(H_{I}^{n-i}(R)\right)$, we recover Lyubeznik's result:

Corollary 3.4. Let $A$ be a ring that admits a surjective ring homomorphism $\pi$ : $R \longrightarrow A$, where $R=k\left[\left[x_{1}, \ldots, x_{n}\right]\right]$ is the formal power series ring. Set $I=\operatorname{ker} \pi$ and let $\mathfrak{p} \subseteq A$ be a prime ideal. The Bass numbers $\mu_{p}\left(\mathfrak{p}, H_{I}^{n-i}(R)\right)$ depend only on $A, \mathfrak{p}, p$ and $i$ but not on $R$ or $\pi$.

When $\mathfrak{p}$ is the zero ideal, we obtain the invariance with respect to $R / I$ of the multiplicities of the characteristic cycle of $H_{I}^{n-i}(R)$.

Corollary 3.5. Let $A$ be a local ring that admits a surjective ring homomorphism $\pi: R \longrightarrow A$, where $R=k\left[\left[x_{1}, \ldots, x_{n}\right]\right]$ is the formal power series ring. Set $I=\operatorname{ker} \pi$ and let

$$
C C\left(H_{I}^{n-i}(R)\right)=\sum m_{i, \alpha} V_{\alpha}
$$

be the characteristic cycle of the local cohomology modules $H_{I}^{n-i}(R)$. Then the multiplicities $m_{i, \alpha}$ depend only on $A, i$ and $\alpha$ but not on $R$ or $\pi$.

Collecting these multiplicities by the dimension of the corresponding irreducible varieties we define the following invariants:

Definition 3.6. Let $I \subseteq R$ be an ideal. If $C C\left(H_{I}^{n-i}(R)\right)=\sum m_{i, \alpha} V_{\alpha}$ is the characteristic cycle of the local cohomology modules $H_{I}^{n-i}(R)$, then we define

$$
\gamma_{p, i}(R / I):=\left\{\sum m_{i, \alpha} \mid \operatorname{dim}\left(\pi\left(V_{\alpha}\right)\right)=p\right\}
$$

One may prove that these invariants have the same properties as Lyubeznik numbers (see [9, Section 4]). Namely, let $d=\operatorname{dim}(R / I)$. Then $\gamma_{p, i}(R / I)=0$ if $i>d, \gamma_{p, i}(R / I)=0$ if $p>i$ and $\gamma_{d, d}(R / I) \neq 0$. In particular, we can collect them in a triangular matrix that we will denote by $\Gamma(R / I)$. We point out that these invariants are finer than the Lyubeznik numbers.

Example 3.7. Let $R=k\left[\left[x_{1}, x_{2}, x_{3}, x_{4}, x_{5}\right]\right]$. Consider the ideals

- $I_{1}=\left(x_{1}, x_{2}, x_{5}\right) \cap\left(x_{3}, x_{4}, x_{5}\right)$;

- $I_{2}=\left(x_{1}, x_{2}, x_{5}\right) \cap\left(x_{3}, x_{4}, x_{5}\right) \cap\left(x_{1}, x_{2}, x_{3}, x_{4}\right)$.

The characteristic cycle of the corresponding local cohomology modules can be computed by means of [1, Theorem 3.8]. Collecting the multiplicities we obtain the triangular matrices

$$
\Gamma\left(R / I_{1}\right)=\left(\begin{array}{lll}
0 & 1 & 0 \\
& 0 & 0 \\
& & 2
\end{array}\right), \quad \Gamma\left(R / I_{2}\right)=\left(\begin{array}{lll}
0 & 2 & 0 \\
& 1 & 0 \\
& & 2
\end{array}\right) .
$$


Computing the Lyubeznik numbers (see [1, Theorem 4.4]), we obtain the triangular matrix

$$
\Lambda\left(R / I_{1}\right)=\Lambda\left(R / I_{2}\right)=\left(\begin{array}{lll}
0 & 1 & 0 \\
& 0 & 0 \\
& & 2
\end{array}\right) .
$$

We have to point out that the quotient ring $R / I_{1}$ is Buchsbaum, but $R / I_{2}$ is not.

Remark 3.8. In order to compute the Lyubeznik numbers $\lambda_{p, i}(R / I)$ for a given ideal $I \subseteq R$ and arbitrary $i, p$ we have to refer to U. Walther's algorithm [11]. When $I$ is a squarefree monomial ideal, a description of these invariants is given in [1] and [13]. Some other particular computations may also be found in [6], [7, 8] and 12]. The multiplicities of the characteristic cycle of $H_{\mathfrak{p}}^{p}\left(H_{I}^{n-i}(R)\right)$, where $I$ is a squarefree monomial ideal and $\mathfrak{p}$ is any homogeneous prime ideal, have been computed in [2].

When $I$ is a squarefree monomial ideal (resp. the defining ideal of an arrangement of linear varieties), the multiplicities of the characteristic cycle of $H_{I}^{n-i}(R)$ have been computed in [1] (resp. [3]).

\section{ACKNOWLEDGMENTS}

I would like to thank my advisor Santiago Zarzuela for his invaluable assistance in writing this paper and the referee for many helpful suggestions.

\section{REFERENCES}

1. J. Àlvarez Montaner, Characteristic cycles of local cohomology modules of monomial ideals, J. Pure Appl. Algebra 150 (2000), 1-25. MR 2001d:13016

2. J. Àlvarez Montaner, Local cohomology modules supported on monomial ideals, Ph.D. Thesis, Univ. Barcelona, 2002.

3. J. Àlvarez Montaner, R. García López, and S. Zarzuela, Local cohomology, arrangements of subspaces and monomial ideals, Adv. in Math. 174 (2003), 35-56.

4. J. E. Björk, Rings of differential operators, North-Holland Mathematical Library, Vol. 21, Amsterdam, 1979. MR 82g:32013

5. S. C. Coutinho, A primer of algebraic $\mathcal{D}$-modules, London Mathematical Society Student Texts, Cambridge University Press, 1995. MR 96j:32011

6. R. Garcia and C. Sabbah, Topological computation of local cohomology multiplicities, Collect. Math. 49 (1998), 317-324. MR 2000a:13029

7. K. I. Kawasaki, On the Lyubeznik number of local cohomology modules, Bull. Nara Univ. Ed. Natur. Sci. 49 (2000), 5-7. MR 2001m:13024

8. K. I. Kawasaki, On the highest Lyubeznik number, Math. Proc. Cambridge Philos. Soc. 132 (2002), 409-417. MR 2003b:13026

9. G. Lyubeznik, Finiteness properties of local cohomology modules, Invent. Math. 113 (1993), 41-55. MR 94e: 13032

10. Z. Mebkhout, Le formalisme des six opérations de Grothendieck pour les $\mathcal{D}_{X}$-modules cohérents, Travaux en Cours, Vol. 35, Hermann, Paris, 1989. MR 90m:32026

11. U. Walther, Algorithmic computation of local cohomology modules and the cohomological dimension of algebraic varieties, J. Pure Appl. Algebra 139 (1999), 303-321. MR 2000h:13012

12. U. Walther, On the Lyubeznik numbers of a local ring, Proc. Amer. Math. Soc. 129(6) (2001), 1631-1634. MR 2001m:13026

13. K. Yanagawa, Bass numbers of local cohomology modules with supports in monomial ideals, Math. Proc. Cambridge Philos. Soc. 131 (2001), 45-60. MR 2002c:13037

Departament de Matemàtica Aplicada I, Universitat Politècnica de Catalunya, Avinguda Diagonal 647, Barcelona 08028, Spain

E-mail address: Josep.Alvarez@upc.es 